\title{
Die Alterssicherung von Beamten in Slowenien
}

\author{
Grega Strban
}

I. Einleitung

II. Der öffentliche Dienst im slowenischen Rechtssystem

III. Die Rechtsstellung der Beschäftigten im öffentlichen Dienst

1. Verfassungsrechtliche Vorgaben

2. Die monistische Konzeption des Arbeitsrechts

3. Allgemeine Grundsätze des Beamtensystems

4. Das Beamtenverhältnis
a) Abschluß eines Dienstvertrages
b) Inhalt des Dienstvertrags
c) Auflösung des Dienstvertrags
d) Rechtsweg

IV. Die Altersversorgung von Beamten

1. Das Verfassungsrecht

2. Die verschiedenen Altersvorsorgesysteme für Beamte

a) Die gesetzliche Rentenversicherung

aa) Allgemeiner Geltungsbereich

bb) Leistungen

cc) Finanzierung und Verwaltung

b) Die obligatorische Alterszusatzversicherung

c) Die freiwillige Alterszusatzversicherung

d) Die kollektive Alterszusatzversicherung für Beamte

e) Die staatliche Rente

V. Abschließende Bemerkungen und die weitere Entwicklung de lege ferenda 


\section{Einleitung}

Die unterschiedliche rechtliche Stellung von Beschäftigten im öffentlichen Dienst und Arbeitnehmern im privaten Sektor geht auf die absolutistische Staatsauffassung zurück. Beschäftigte des öffentlichen Dienstes standen in keinem Arbeitsverhältnis zum Staat. Vielmehr wurde ein besonderes persönliches Dienstverhältnis mit dem Kaiser (Herrscher) begründet, ${ }^{1}$ das sich durch eine uneingeschränkte Loyalität gegenüber dem Herrscher auszeichnete. Die höchsten Ämter waren nur Personen mit aristokratischer Herkunft zugänglich. Der Staatsdienst wurde mit der Ausübung hoheitlicher Macht gleichgesetzt, und die Staatsdiener wurden als Träger des staatlichen Machtmonopols angesehen. ${ }^{2}$ Später wurde diese unterschiedliche Behandlung mit der Theorie der Staatssouveränität gerechtfertigt: Der Staat (die Regierung) ist souverän und einzig dem Gesetzgeber unterstellt, selbst wenn er als Arbeitgeber auftritt. Die rechtliche Sonderstellung der Staatsbediensteten wurde mit ihrer Befugnis zur Ausübung hoheitlicher Gewalt begründet. Das zeigte sich nicht nur in ihrem Verhältnis zum Dienstherrn, ${ }^{3}$ sondern auch in der Sonderbehandlung bei der Absicherung gegen bestimmte soziale Risiken, z.B. Krankheit und Alter.

Grundsätzlich stellt sich die Frage, ob eine wesentlich unterschiedliche Stellung der Beschäftigten im öffentlichen Dienst im Zuge des gegenwärtigen demokratischen Verständnisses von der Gleichbehandlung aller Menschen und Bürger noch tragbar ist. ${ }^{4}$

1 Die monarchische Staatsgewalt erfuhr keinerlei Einschränkung durch andere Institutionen wie Kirchen, Gesetzgeber oder gesellschaftliche Eliten. Siehe dazu umfassend Anderson, Lineages of the Absolulist State, 1974.

2 Virant, Pravna ureditev javne uprave (Rechtliche Gestaltung der Öffentliche Verwaltung), 2002, S. 187.

3 Das Dienstverhältnis wurde einseitig vom Dienstherrn geprägt. Die Rechtsstellung der Beamten war eher dem Verwaltungsrecht als dem modernen Arbeitsrecht zuzuordnen. Bestimmte Rechte, wie z.B. die gewerkschaftliche Organisierung, waren eingeschränkt. Dafür gewährte der Staat eine bevorzugte Behandlung bei der Entlohnung und der Arbeitsplatzsicherheit. Vgl. Končar, Uresničevanje sindikalne svobode in pravice do stavke javnih uslužbencev (Verwirklichung der Gewerkschaftsfreiheit und das Streikrecht der Beamten), in: Pavčnik/Polajnar-Pavčnik/Wedam-Lukić (Hrsg.), Temeljne pravice, 1997, S. 279.

4 So bestimmt die französische Déclaration des droits de l'Homme et du Citoyen von 1789, daß „La garantie des droits de l'homme et du citoyen nécessite une force publique; cette force est donc instituée pour l'avantage de tous, et non pour l'utilité particulière de ceux à qui elle est confiée“, Art. 12. Auch in der Weimarer Verfassung von 1919 steht geschrieben: „Alle Staatsbürger ohne Unterschied sind nach Maßgabe der Gesetze und entsprechend ihrer Befähigung und ihren Leistungen zu den öffentlichen Ämtern zuzulassen, Ruhegehalt und Hinterbliebenenversorgung werden gesetzlich geregelt, die Beamten sind Diener der Gesamtheit, nicht einer Partei“, Art. 128-130. 
Auch ist dies nicht die effizienteste Art der Staatsverwaltung in einer modernen Gesellschaft. ${ }^{5}$

Die Beschäftigung im öffentlichen Dienst wird heute als Tätigkeit im öffentlichen Interesse verstanden. Die Stellung der Staatsdiener hat im Laufe der Zeit Änderungen erfahren, da ihre Privilegien verringert wurden, was auch auf die Ausweitung des öffentlichen Sektors zurückzuführen ist. Ihnen werden arbeitsrechtliche Ansprüche zugestanden, die zuvor nur Arbeitnehmer geltend machen konnten. ${ }^{6}$ Darüber hinaus wird die soziale Sicherung zunehmend der von Arbeitnehmern angenähert.

Es bestehen weiterhin Unterschiede, die nicht in dem Rechtsstatus des Staates (oder der Kommunen) als Dienstherr begründet sind, sondern sich aus der besonderen Natur des öffentlichen Dienstes ergeben. Die Tätigkeit im öffentlichen Dienst prägt nicht nur die Organisation, innerhalb derer sie erbracht wird (ein Ministerium z.B.), sondern hat weitreichendere Auswirkungen, wenn das Wohl der Allgemeinheit betroffen ist.

Der vorliegende Beitrag geht in erster Linie der Frage nach, ob sich die dienstrechtliche Stellung der Beamten derart von der der Arbeitnehmer unterscheidet, daß eine Sonderbehandlung im Bereich der Alterssicherung gerechtfertigt ist. Dazu müssen der Beamtenbegriff erläutert, der Aufgabenbereich dargestellt und die Sonderbestimmungen im slowenischen Alterssicherungssystem untersucht werden.

\section{Der öffentliche Dienst im slowenischen Rechtssystem}

In der slowenischen Rechtsordnung werden Beschäftigte im öffentlichen Dienst in verschiedene Kategorien eingeteilt.

Zunächst wird zwischen „Funktionären/Trägern öffentlicher Befugnisse“ (funkcionarji) und „Beamten“ (javni uslužbenci) unterschieden. Bei den Beamten wird wiederum unterschieden zwischen solchen Beamten, die öffentliche Aufgaben erfüllen (uradniki) und Beamten, die als Fachexperten darüber hinausgehende Dienste leisten (strokovno-tehnični uslužbenci).

Funkcionarji werden (direkt oder indirekt) gewählt bzw. nach politischen Kriterien für den staatlichen oder kommunalen Bereich ernannt. Ihre Rechtsstellung wird in zwei allgemeinen Gesetzen geregelt: zum einen im Gesetz über Funktionäre in der staatlichen Verwaltung (Zakon o funkcionarjih $v$ državnih organih $-Z F D O)^{7}$ und zum anderen im

5 Bučar, Uslužbenci v javni upravi: nekatera načelna izhodišča za zakonsko ureditev (Beamte in der Öffentlichen Verwaltung: Einige grundsätzliche Ausgangspunkte für die gesetzliche Regelung), 1999, S. 180, 182.

6 Zudem erschwerte die zunehmende Privatisierung im öffentlichen Sektor die Abgrenzung zwischen Beamten und Arbeitnehmern. Vgl. Končar, in: Pavčnik/Polajnar-Pavčnik/Wedam-Lukić (Hrsg.), Temeljne pravice (Fußn. 3), S. 280.

7 Amtsblatt der Republik Slowenien (RS), Nr. 30/90, zuletzt geändert 1993. 
Gesetz über die kommunale Selbstverwaltung (Zakon o lokalni samoupravi - ZLS). ${ }^{8}$ Für bestimmte Gruppen von staatlichen funkcionarji gelten Sondergesetze. Zu diesen Gruppen gehören z.B. Parlamentsabgeordnete (Nationalversammlung und Nationalrat), ${ }^{9}$ der Präsident der Republik, ${ }^{10}$ der Premierminister, die Minister und die Staatssekretäre, ${ }^{11}$ Richter an ordentlichen Gerichten und am Verfassungsgericht, ${ }^{12}$ Staatsanwälte ${ }^{13}$ sowie die Mitglieder des Rechnungshofes. ${ }^{14} \mathrm{Zu}$ den funkcionarji in den Gemeinden zählen die Mitglieder des Gemeinde-/Stadtrats, der Bürgermeister und seine Vertreter. ${ }^{15} \mathrm{Im}$ Allgemeinen üben funkcionarji ihr Amt berufsmäßig aus. Ausnahmen bilden beispielsweise Mitglieder des Nationalrats, Gemeinde- oder Stadtratsmitglieder und Bürgermeister. Letztere können entscheiden, ob sie das Amt berufsmäßig oder ehrenamtlich ausüben wollen. Die Amtszeit ist befristet und richtet sich nach der Legislaturperiode oder dem Tag der Ernennung (Mandat). Eine Sonderstellung nehmen Richter und Staatsanwälte an ordentlichen Gerichten ein, die wegen des Erfordernisses der Objektivität und Unbefangenheit für eine unbefristete Amtszeit gewählt werden, wobei die Amtszeit unter bestimmten Voraussetzungen beendet werden kann. Funkcionarji und Beamte haben unterschiedliche Aufgaben. Die Rolle der Ersteren ist politischer Natur, eine Fachkompetenz ist nicht erforderlich. Sie treffen politische Entscheidungen treffen, deren fachliche Grundlagen von Beamten erarbeitet werden. ${ }^{16}$

Die Rechtsstellung der Beamten dagegen wird in dem Beamtengesetz (Zakon o javnih uslužbencih - ZJU) geregelt ${ }^{17}$, von dessen Anwendungsbereich die funkcionarji nicht erfaßt werden. Dadurch wird die besondere Stellung der Beamten zur Wahrnehmung öffentlicher Aufgaben hervorgehoben.

Als Beamter wird eine Person bezeichnet, die in einem öffentlichen Dienstverhältnis steht. Der öffentliche Sektor umfaßt nicht nur die staatliche und kommunale Verwaltung, sondern auch die Verwaltung öffentlicher Agenturen, öffentlicher Fonds und öf-

8 Amtsblatt RS, Nr. 72/93, zuletzt geändert 2008.

9 Abgeordnetengesetz (Zakon o poslancih-ZPos), Amtsblatt RS, Nr. 48/92, zuletzt geändert 2008. Gesetz über den Nationalrat (Zakon o državnem svetu - ZDSve), Amtsblatt RS, Nr. 44/92, zuletzt geändert 2005.

10 Gesetz über die Amtsausübung als Präsident der Republik (Zakon o zagotavljanju pogojev za opravljanje funkcije predsednika republike - ZZPOFPR), Amtsblatt RS, Nr. 15/03.

11 Gesetz über die Regierung der Republik Slowenien (Zakon o Vladi Republike Slovenije - ZVRS), Amtsblatt RS, Nr. 4/93, zuletzt geändert 2008. Verwaltungsgesetz (Zakon o državni upravi - ZDU1) Amtsblatt RS, Nr. 52/02, zuletzt geändert 2005.

12 Gesetz über das Gerichtsverfahren (Zakon o sodniški službi-ZSS), Amtsblatt RS, Nr. 19/94, zuletzt geändert 2007. Verfassungsgerichtsgesetz (Zakon o ustavnem sodišču - ZUstS), Amtsblatt RS, Nr. 63/94, zuletzt geändert 2007.

13 Gesetz über die Staatsanwaltschaft (Zakon o državnem tožilstvu - ZDT), Amtsblatt RS, Nr. 15/94, zuletzt geändert 2007.

14 Rechnungshofgesetz (Zakon o računskem sodišču - ZRacS-1), Amtsblatt RS, Nr. 11/01.

15 Art. 34.b ZLS.

16 Virant, Pravna ureditev javne uprave (Fußn. 2), S. 193.

17 Amtsblatt RS, Nr. 56/02, zuletzt geändert 2008. 
fentlicher Einrichtungen, wie z.B. das slowenische Alters- und Invaliditätsversicherungsinstitut, sowie jede andere juristische Person des öffentlichen Rechts, wenn sie direkt oder indirekt aus dem Staatshaushalt oder dem kommunalen Haushalt finanziert werden. Ferner gibt es Gesetze, die besondere Bestimmungen für bestimmte Beamtengruppen enthalten, wie die Polizei, ${ }^{18}$ die Gerichtspolizei (Justizvollzugsbeamte), ${ }^{19}$ die slowenische Armee, ${ }^{20}$ Zollbeamte $^{21}$ und Inspektoren ${ }^{22}$.

Die Rechtsstellung von Beamten, die im sogenannten öffentlichen Dienstleistungssektor bei einer juristischen Person des öffentlichen Rechts beschäftigt sind, wie Ärzte, Lehrer, Postbeamte, wird in diesem Beitrag nicht näher untersucht. Dies entspricht der Rechtsprechung des EuGH. Demzufolge umfaßt der „öffentliche Dienst“ nur Ämter, die eine unmittelbare oder mittelbare Teilnahme an der Ausübung hoheitlicher Befugnisse und an der Wahrnehmung solcher Aufgaben mit sich bringen, die auf die Wahrung der allgemeinen Belange des Staates oder anderer öffentlicher Stellen gerichtet sind. Das Kriterium zur Abgrenzung ist gemäß dem EuGH die inhaltliche Ausgestaltung des Amtes und nicht die rechtliche Qualifikation des Beschäftigungsverhältnisses. ${ }^{23}$

\section{Die Rechtsstellung der Beschäftigten im öffentlichen Dienst}

\section{Verfassungsrechtliche Vorgaben}

Die slowenische Verfassung unterscheidet in ihrem Abschnitt über die Staatsorganisation zwischen „Trägern öffentlicher Befugnisse“ und „Beschäftigten im Verwaltungsdienst“. Beispielsweise ist die Wahl der Parlamentsabgeordneten, des Präsidenten der Republik, des Premierministers, der Minister und der Richter als Träger öffentlicher Befugnisse verfassungsrechtlich, die Ernennung von Beamten dagegen gesetzlich geregelt (Art. 120).

18 Polizeigesetz (Zakon o policiji - ZPol), Amtsblatt RS, Nr. 49/98, zuletzt geändert 2009.

19 Gesetz über den Strafvollzug (Zakon o izvrševanju kazenskih sankcij - ZIKS-1) Amtsblatt RS, Nr. 22/00, zuletzt geändert 2009.

20 Verteidigungsgesetz (Zakon o obrambi - ZObr), Amtsblatt RS, Nr. 82/94, zuletzt geändert 2004. Gesetz über den Wehrdienst in der Slowenischen Armee (Zakon o službi v Slovenski vojski ZSSloV), Amtsblatt RS, Nr. 68/07.

21 Gesetz über den Zolldienst (Zakon o carinski službi - ZCS-1), Amtsblatt RS, Nr. 56/99, zuletzt geändert 2009.

22 Inspektionsgesetz (Zakon o inšpekcijskem nadzoru - ZIN) Amtsblatt RS, Nr. 56/02, zuletzt geändert 2007. Neben dem Inspektionsgesetz als lex generalis gibt es Sondergesetze, die z.B. Arbeits-, Gesundheits- oder Marktinspektionen regeln.

23 EuGH, Rs. C-66/85, Slg. 1986, 2121 - Lawrie-Blum; EuGH, Rs. C-152/73, Slg. 1974, 153 - Sotgiu; Blanpain, European Labour Law, 2006, S. 335. 
Die Hierarchie zwischen politischen Trägern öffentlicher Befugnisse und Beamten muß vom Gesetzgeber bestimmt werden. Dabei muß der Gesetzgeber den Beamten das $\mathrm{Ma}$ an Unabhängigkeit gewährleisten, das eine unabhängige Verwaltung ermöglicht. ${ }^{24}$ Ein wesentliches Kriterium für eine unabhängige Dienstausübung ist ein stabiles Dienstverhältnis zwischen dem Beamten und seinem Dienstherrn. ${ }^{25}$

Der Eintritt in den Verwaltungsdienst erfordert nach der Verfassung - außer in den gesetzlich geregelten Fällen (Art. 122) - eine öffentliche Ausschreibung. Die Tätigkeiten in der öffentlichen Verwaltung sind solche, die die Ausübung hoheitlicher Gewalt vorsehen, d.h. den Erlaß allgemeiner Regelungen und die Entscheidungsfindung im Einzelfall, können aber weder auf den gesamten öffentlichen Dienst noch auf die verwaltungstechnischen Aufgaben der Verwaltungsorgane ausgedehnt werden.

Das Erfordernis einer öffentlichen Ausschreibung verfolgt das Ziel, jedem Bewerber die gleichen Zugangschancen zum öffentlichen Dienst unter gleichen Voraussetzungen einzuräumen, ohne einen bestimmten Bewerber unbegründet zu bevorzugen. Der gleiche Zugang zu öffentlichen Ämtern wird auch durch Artikel 49 der Verfassung gesichert. Diese Verfassungsnorm gewährleistet die Freiheit der Arbeit, enthält jedoch keine Garantie für die Dauer der Beschäftigung oder für eine dauerhafte Beschäftigung an einem bestimmten Arbeitsplatz. ${ }^{26}$ Zudem ist das Recht auf Arbeitsfreiheit Einschränkungen unterworfen, wenn bestimmte Tätigkeiten nicht mit dem öffentlichen Dienst vereinbar sind. ${ }^{27}$ Schließlich soll der jeweils am besten geeignete Bewerber eingestellt werden.

Ausnahmen von einer öffentlichen Ausschreibung müssen in Übereinstimmung mit dem Grundsatz der Verhältnismäßigkeit gesetzlich geregelt werden. ${ }^{28}$ Darüber hinaus muß der verfassungsrechtliche Gleichheitssatz (Art. 14) berücksichtigt werden. Ungleiche Behandlung von Arbeitnehmern und Beamten in Angelegenheiten der Beschäftigung oder im Rahmen von Arbeits- und Dienstverhältnissen sind grundsätzlich untersagt. ${ }^{29}$ Bei der Regelung des Beamtenrechts können zudem auch andere verfassungsrechtliche Bestimmungen von Bedeutung sein. Zu nennen sind beispielsweise die Koalitionsfreiheit (Art. 76) und das Streikrecht (Art. 77), wobei das Streikrecht je nach Art der Tätigkeit im Interesse der Allgemeinheit eingeschränkt werden kann. Das Mitbestimmungsrecht von Arbeitnehmern ist dagegen auf die Verwaltung von Wirtschaftsunternehmen und Anstalten beschränkt (Art. 75).

24 Art. 120 der Slowenischen Verfassung normiert in Absatz 2, daß die Verwaltungsorgane ihre Aufgaben selbstständig im Rahmen und auf der Grundlage der Verfassung und der Gesetzte ausüben.

25 Entscheidung des Verfassungsgerichts Nr. U-I-90/05 vom 7.7.2005, OdIUS XIV, 66.

26 Kresal, in: Šturm u.a. (Hrsg.), Komentar Ustave Republike Slovenije (Kommentar der Verfassung von der Republik Slowenien), 2002, S. 509.

27 Entscheidung des Verfassungsgerichts No. U-I-329/04, 12.5.2005, OdIUS XIV, 27.

28 Virant, in: Šturm u.a. (Hrsg.), Komentar Ustave Republike Slovenije (Fußn. 26), S. 867, 893,

29 Bohinc, Delovna in uslužbenska razmerja (Arbeits- und Beamtenverhältnisse), 2004, S. 65. 


\section{Die monistische Konzeption des Arbeitsrechts}

Im heutigen Slowenien unterlag die Rechtsstellung der Beamten vor dem 2. Weltkrieg anderen Regelungen als die der Arbeitnehmer. ${ }^{30}$ Seit 1957 wurde der Grundsatz der Uniformität aller Beschäftigungsverhältnisse in den Vordergrund gestellt, mit der Folge, daß jedes Gesetz, das die Beschäftigungsverhältnisse regelt, die Eigenschaft eines lex generalis hat und grundsätzlich für Beschäftigungsverhältnisse aller Beschäftigten im öffentlichen wie auch privaten Sektor Geltung beansprucht. Besonderheiten mancher Tätigkeiten erfordern allerdings eine gesonderte Regelung in einzelnen Gesetzen. 31

Die monistische Konzeption des Arbeitrechts schien mit dem letzten Gesetz über die Beschäftigungsverhältnisse (Zakon o delovnih razmerjih - ZDR) von $2002^{32}$ und dem Beamtengesetz (ZJU) geschwächt oder sogar de facto außer Kraft gesetzt worden zu sein. Es gab Literaturstimmen, die für eine strikte dualistische Regelung der Arbeitsverhältnisse im öffentlichen und privaten Sektor eintraten. Als Argumente wurden vorgebracht, daß es für die Einführung von vertraglichen Komponenten keinen besonderen Grund gäbe, der Inhalt eines Arbeitsvertrages größtenteils im Voraus festgelegt werde sowie die Verhandlungs- und Einigungsmöglichkeiten beschränkt seien. ${ }^{33}$

Der Gesetzgeber entschied sich jedoch, die monistische Konzeption des Arbeitsrechts zumindest de jure aufrechtzuerhalten. Es wurde angeführt, daß die Entwicklung des Arbeitsrechts insbesondere einiger europäischer Staaten Berücksichtigung finden müsse. Der Trend scheint dahin zu gehen, die Rechtsstellung einer breiten Arbeitnehmerschicht unabhängig von ihrem Beschäftigungssektor einheitlich zu regeln. ${ }^{34}$ Darüber hinaus bedarf jede Beschäftigung ihrer Natur nach einer vertraglichen Grundlage. Es ist nicht möglich, ein Beschäftigungsverhältnis durch Verwaltungshandeln zu begründen. ${ }^{35}$ Folglich findet das $Z D R$ als lex generalis Anwendung, wenn besondere Regelungen fehlen. Spezielle Vorschriften enthält das $Z J U$, das die Dienstverhältnisse der Beamten ausführlich regelt, und ist daher in Bezug auf das $Z D R$ - ebenso wie auch weitere Sondergesetze für bestimmte Beamten und das Gesetz über die Entlohnung im öffentlichen Sektor ${ }^{36}$ - lex specialis.

30 So z.B. im Gesetz über die Träger öffentlicher Befugnisse und anderer Beamten im Königreich der Serben, Kroaten und Slowenien (SHS), Ilić, 1927. Zum Gesetz über die Beamten im Königreich Yugoslawien (1931 erlassen), siehe Bohinc (Fußn. 29), S. 23.

31 Končar, in: Pavčnik/Polajnar-Pavčnik/Wedam-Lukić (Hrsg.), Temeljne pravice (Fußn. 3), S. 33.

32 Amtsblatt RS, Nr. 42/02, zuletzt geändert 2007.

33 Pirnat, Nekatera vprašanja pravnega položaja javnih uslužbencev (Einige Fragen der Rechtslage der Beamten), Podjetje in delo, Nr. 6-7/2001, S. 1349, 1353.

34 Končar, in: Pavčnik/Polajnar-Pavčnik/Wedam-Lukić (Hrsg.), Temeljne pravice (Fußn. 3), S. 33.

35 Pirnat (Fußn. 33), Podjetje in delo, Nr. 6-7/2001, S. 1349, 1353.

36 Gesetz über die Entlohnung im Öffentlichen Sektor (Zakon o sistemu plač v javnem sektorju ZSPJS), Amtsblatt RS, Nr. 56/02, zuletzt geändert 2007. 
Die besonderen Regelungen für Beamte beruhen nicht nur auf verfassungsrechtlichen Vorgaben, sondern auch auf allgemeinen Grundsätzen, die im ZJU verankert sind.

\section{Allgemeine Grundsätze des Beamtensystems}

Das ZJU besteht aus zwei Teilen. Im ersten Teil werden der Beamtenbegriff definiert und einige allgemeine Grundsätze geregelt, die auf alle Beamte Anwendung finden. Der zweite Teil enthält Sonderbestimmungen für Beamte, die in der staatlichen und kommunalen Verwaltung tätig sind, sowie einige zusätzliche Grundsätze.

$\mathrm{Zu}$ den allgemeinen Grundsätzen, die für alle Beamten gelten, gehört der Grundsatz des gleichen Zugangs zu öffentlichen Ämtern. Jeder hat unter gleichen Bedingungen gleichen Zugang zu jeder Art von Beschäftigung im öffentlichen Dienst. Dieser Grundsatz basiert auf den bereits erwähnten verfassungsrechtlichen Bestimmungen der Artikel 14 und 49. Das Hauptkriterium bei der Wahl eines Bewerbers ist die fachliche Qualifikation. Dennoch hat der Gesetzgeber kein wirklich offenes System des Zugangs zu einem öffentlichen Amt geschaffen. Zuerst müssen „,interne Reserven“ ausgeschöpft werden, bevor eine öffentliche Ausschreibung erfolgt. Daher ist eine Mischform aus der Verknüpfung eines offenen Systems freier Stellen mit einem Beförderungssystem für den Zugang zu einem öffentlichen Amt kennzeichnend. ${ }^{37}$

Das Prinzip der Legalität verhindert willkürliche Entscheidungen. Ein Beamter darf keine öffentlichen Aufgaben ohne gesetzliche Grundlage ausführen. Daher muß er sogar gesetzwidrige Anordnungen zurückweisen. Rechtsicherheit und Objektivität müssen gewahrt werden.

Das Prinzip der Professionalität verpflichtet Beamte zur fachlich kompetenten, gewissenhaften und fristgerechten Wahrnehmung öffentlicher Aufgaben. Sie sollen an Fortbildungen und Schulungen teilnehmen, die vom Dienstherrn anzubieten sind. Dies soll den Wettbewerb unter den Beamten fördern. ${ }^{38}$

Die Einhaltung ethischer Normen schreibt die Pflicht zu achtungswürdigem, ehrenhaftem Verhalten vor. Die Regierung hat einen besonderen Verhaltenskodex für Beamte ${ }^{39}$ und einen Ethikkodex für Träger politischer Ämter erlassen, den Bewerber für ein bestimmtes Amt unterzeichnen müssen. Die detaillierten Regelungen über die Annahme von Geschenken verbieten Beamten bei der Erfüllung öffentlicher Aufgaben, Geschenke von hohem Wert anzunehmen, 40 um Korruption zu verhindern und die unparteiliche Entscheidungsfindung zu gewährleisten.

37 Virant, in: Bugarič, u.a. (Hrsg.), Komentar zakonov s področja uprave (Kommentar der Verwaltungsgesetze), 2004, S. 65.

38 Virant, a.a.O., S. 71.

39 Amtsblatt RS, Nr. 8/01.

40 Nach dem Gesetz über die Verhinderung der Korruption dürfen Amtsträger keinerlei Geschenke annehmen (Zakon o preprečevanju korupcije - ZPKor, Amtsblatt RS, Nr. 2/2004). 
Die Verpflichtung zur Amtsverschwiegenheit (Geheimhaltungsgrundsatz) hat zur Folge, daß der Beamte auch nach Beendigung seines Dienstverhältnisses zur Geheimhaltung aller offiziellen Daten verpflichtet ist.

Die persönliche Verantwortlichkeit für die getroffenen Entscheidungen soll Beamte dazu anhalten, bei der Erfüllung öffentlicher Aufgaben auf Qualität, zeitnahe Erledigung und Effizienz zu achten.

Das Wirtschaftlichkeitsgebot ist aus dem privaten Sektor übernommen und entspricht der neuen Vorstellung von öffentlicher Verwaltung. ${ }^{41}$

Die rechtliche Unterstützung in straf- und zivilrechtlichen Verfahren folgt aus dem Prinzip zur Wahrung der Berufsinteressen, wenn der Dienstherr die Erfüllung der betreffenden öffentlichen Aufgabe als rechtmäßig bewertet hat.

Der zweite Teil des ZJU sieht weitere Prinzipien für Beamte in staatlichen und kommunalen Verwaltungen vor, die hauptsächlich öffentliche Aufgaben wahrnehmen. ${ }^{42}$ Das Erfordernis der öffentlichen Ausschreibung bei der Besetzung öffentlicher Ämter gehört zu den verfassungsrechtlichen Grundsätzen, die im ZJU näher ausgeführt sind. Die Prinzipien der politischen Neutralität und der Unparteilichkeit verpflichten die Beamten zur Erfüllung öffentlicher Aufgaben im Interesse der Allgemeinheit. Die Förderung der besten Beamten ermöglicht das Leistungsprinzip. Beamte und Arbeitnehmer, die untergeordnete Aufgaben erfüllen, haben keinen Anspruch auf die dauerhafte Beschäftigung auf einer bestimmten Stelle. Vielmehr soll die Möglichkeit von Versetzungen die Mobilität innerhalb einer Behörde oder zwischen staatlichen und/oder kommunalen Behörden ermöglichen. Das Öffentlichkeitsprinzip ist Ausdruck einer umfassenderen Verwaltungstransparenz. Es definiert das Verhältnis zwischen Verwaltung und Bürger neu, weil die Öffentlichkeit über die Tätigkeiten und Maßnahmen des öffentlichen Dienstes informiert werden soll. Ein Sondergesetz regelt den Zugang zu öffentlichen Dokumenten. ${ }^{43}$

\section{Das Beamtenverhältnis}

Das Beamtenverhältnis in Slowenien ist im Vergleich zu einigen anderen europäischen Staaten kein besonderes öffentlich- oder verwaltungsrechtliches Dienstverhältnis zwischen dem Beamten und dem Staat bzw. der Kommune als Dienstherr. ${ }^{44}$ Bei Abschluß des Dienstvertrages übt der Staat gegenüber dem Beamten keine hoheitliche

41 Die Übernahme von Grundsätzen aus dem privaten Sektor können sich bei Anwendung auf den öffentlichen Sektor in gewisser Weise als problematisch erweisen, Virant, in: Bugarič, u.a. (Hrsg.), Komentar zakonov s področja uprave (Fußn. 37), S. 82.

42 Art. 27-32 ZJU.

43 Zakon o dostopu do informacij javnega značaja, Amtsblatt RS, Nr. 24/03, zuletzt geändert 2006.

44 Virant, in: Bugarič, u.a. (Hrsg.), Komentar zakonov s področja uprave (Fußn. 37), S. 87. 
Gewalt aus, sondern beide Parteien stehen als Vertragspartner auf gleicher Ebene. ${ }^{45}$ Der Dienstvertrag ist als Arbeitsvertrag zu qualifizieren ${ }^{46}$, der im $Z D R$ und teilweise im Zivilrecht geregelt wird. ${ }^{47}$ Dessen ungeachtet enthalten der $Z J U$ und einige Sondergesetze besondere Regelungen in Bezug auf Abschluß, Inhalt, Kündigung und gerichtliche Überprüfung von Dienstverträgen.

\section{a) Abschluß eines Dienstvertrages}

Das Verfahren bei Abschluß eines Dienstvertrages ist, je nach dem, ob es sich um einen Beamten oder einen fachlich qualifizierten Arbeitnehmer handelt, unterschiedlich. Für Letzteren finden die allgemeinen Regelungen des Arbeitsrechts Anwendung. ${ }^{48}$

Vor Begründung eines Dienstverhältnisses mit einem Beamten in der staatlichen oder kommunalen Verwaltung muß eine interne Ausschreibung erfolgen. Die ausgeschriebene Stelle kann mit Beamten aus derselben oder einer anderen Behörde, sogar aus der staatlichen und kommunalen Verwaltung besetzt werden. Unter bestimmten Voraussetzungen (wie einer gleichwertigen Stelle, einem gleichen Titel, etc.) kann eine Versetzung selbst gegen den Willen des Beamten vorgenommen werden. 49

Führt die interne Stellenausschreibung nicht zu der Besetzung der ausgeschriebenen Stelle, dann hat eine öffentliche Ausschreibung zu erfolgen. Der Bewerber mit der besten beruflichen Qualifikation wird durch Verwaltungsentscheidung ausgewählt und in das entsprechende Amt ordnungsgemäß berufen. Erst dann wird der Abschluss eines in der Regel unbefristeten - Dienstvertrages angeboten. ${ }^{50}$

Der ausgewählte Bewerber hat einen Anspruch auf den Vertragsabschluss, kann aber selbst nicht zur Unterzeichnung verpflichtet werden. Die Einigung beider Parteien ist erforderlich. ${ }^{51}$ Unterzeichnet der ausgewählte Bewerber den angebotenen Dienstvertrag nicht, dann verliert die Entscheidung über die Berufung in das Amt ihre Rechtsverbindlichkeit. Eine Vorschrift, die den Dienstherrn ermächtigt, den Dienstvertrag nicht zu

45 Keř̌evan, in: Bugarič, u.a. (Hrsg.), Komentar zakonov s področja uprave (Fußn. 37), S. 61.

46 Teilweise wird der Dienstvertrag dem Verwaltungsrecht zugeordnet, auch wenn ein verwaltungsrechtlicher Vertrag als solcher in der slowenischen Rechtsordnung nicht geregelt ist. Als Argument wird vorgetragen, daß der Dienstvertrag eines Beamten im Interesse der Allgemeinheit geschlossen wird und das öffentliche Interesse Auswirkungen auf den Inhalt und den Abschluß des Vertrags hat. Vgl. Pirnat (Fußn. 33), Podjetje in delo, Nr. 6-7/2001, S. 1349, 1355.

47 Allgemeine zivilrechtliche Regelungen können (einschränkend) für den Dienstvertrag Geltung finden, Končar, in: Bečan u.a., Zakon o delovnih razmerjih s komentarjem (Gesetz über die Arbeitsverhältnisse mit Kommentar), 2008, S. 75.

48 Blaha, Sodno varstvo pravic javnih uslužbencev (Gerichtlicher Schutz der Beamtenrechte), Delavci in delodajalci, No. 2-3/2009, S. 359, 367.

49 Sajevec Plavčak, in: Bugarič, u.a. (Hrsg.), Komentar zakonov s področja uprave (Fußn. 37), S. 173.

50 Nur ausnahmsweise kann ein befristeter Dienstvertrag abgeschlossen werden (Art. 68-73 ZJU).

51 Der Dienstvertrag ist nicht Gegenstand der öffentlichen Ausschreibung. Vgl. Pirnat (Fußn. 33), Podjetje in delo, Nr. 6-7/2001, S. 1349, 1355. 
unterzeichnen, nachdem ein Bewerber ausgewählt und in das zu besetzende Amt ernannt wurde, existiert dagegen nicht.

Der Inhalt eines Dienstvertrags wird im ZJU vorgegeben. Eine der wichtigsten Besonderheiten ist, daß der Vertrag den Dienstherrn zur einseitigen Modifikation des Dienstverhältnisses berechtigen muß, weil der Schutz öffentlicher Interessen in bestimmten Fällen die einseitige Änderung eines gegenseitigen Dienstvertrages erfordern kann, 52 denn der Staat hat im Rahmen einer Vertragsbeziehung eine besondere Parteistellung, weil ihm als Träger hoheitlicher Gewalt die volle Vertragsautonomie nicht zusteht, vielmehr diese rechtlich eingeschränkt ist. 53

Für bestimmte Gruppen von Beamten gelten Sonderbestimmungen. Beispielsweise erfordert die Einstellung bei der Polizei, Gerichtspolizei und als Inspektor die Erlangung eines bestimmten Ausbildungsgrades, während für Soldaten eine bestimmte Altersgrenze normiert wird. ${ }^{54}$

\section{b) Inhalt des Dienstvertrags}

Die Vertragsfreiheit bei der inhaltlichen Ausgestaltung von Dienstverträgen ist eingeschränkt.

Unzulässig ist es, Beamten vertraglich mehr Rechte zu gewähren, als in Gesetzen, Verwaltungsvorschriften oder Tarifabschlüssen vorgesehen ist, wenn der Staatskasse hierdurch ein Nachteil entsteht. Im Bereich des Arbeitsrechts dagegen normiert das Gesetz Mindestrechte für Arbeitnehmer, wobei Arbeitnehmer und Arbeitgeber zum Nutzen des Arbeitnehmers andere Vereinbarungen treffen können. ${ }^{55}$

Auch werden Gehälter von Beamten (bis auf bestimmte Zulagen) ${ }^{56}$ in einem Sondergesetz geregelt. Ein Beamter muß zudem Aufgaben erfüllen, die in der Dienstbeschreibung nicht aufgeführt sind, sowie zusätzliche Arbeit leisten und auf Anordnung einer Projektgruppe beitreten.

Ein Beamter, der öffentliche Aufgaben wahrnimmt, darf Tätigkeiten, die mit seiner Position unvereinbar sind, nicht übernehmen. Das ZJU sah umfangreiche Disziplinarstrafen vor. Diese Regelungen wurden 2005 aufgehoben, mit dem Ziel, die Anwendung

52 Beispielsweise kann der Dienstherr einen Dienstvertrag einseitig modifizieren, wenn ein Beamter einer Vertragsänderung bezüglich der Ernennung in ein anderes Amt, Zuordnung in eine höhere Gehaltsgruppe oder der Versetzung auf eine andere Stelle nicht zustimmt (Art. 53 ZJU).

53 Kerševan, Približevanje ureditve položaja javnega uslužbenca položaju delavca v zasebnem sektorju (Annäherung der Rechtslage von Beamten, zu der von Arbeiter im Privatsektor), Delavci in delodajalci, No. 2-3/2005, S. 391, 399.

54 Für Soldaten gilt die Altersgrenze von 25 Jahren; für Offiziere ist eine Altersgrenze von 30 Jahren vorgeschrieben (Art. $88 \mathrm{ZObr}$ ).

55 Končar, in: Bečan u.a., Zakon o delovnih razmerjih s komentarjem (Fußn. 47), S. 56.

56 So haben beispielsweise Soldaten ein Recht auf eine Zulage, wenn sie auf einer Mission außer Landes unterwegs sind (Art. 98.c ZObr). 
allgemeiner arbeitsrechtlicher Regelungen auf Beamte und Arbeitnehmer unter Beachtung des Grundsatzes der Gleichbehandlung zu ermöglichen. ${ }^{57}$

Schließlich werden Arbeitszeit, Pausen, Jahresurlaub, Heim- und Nachtarbeit, Bereitschaftsdienst und Disziplinarstrafen für manche Beamte gesondert geregelt. 58

Der Dienstvertrag als Instrument zur Begründung eines Dienstverhältnisses ermöglicht die Beteiligung von Sozialpartnern im öffentlichen Sektor. Eine Einigung bei der Modifizierung öffentlich-rechtlicher Dienstverhältnisse zu erzielen, ist demokratischer, als diese dem Staat als Träger hoheitlicher Befugnisse zu belassen. ${ }^{59}$ Deshalb haben Beamte einschließlich des Militärs und der Polizei das Recht, sich gewerkschaftlich zu organisieren. Gewerkschaften können für die Rechte der Beamten eintreten, insbesondere über Tarifverträge mit dem Staat die Beamtenbesoldung aktiv beeinflussen. ${ }^{60}$ Beamte haben auch ein in der Verfassung verankertes Streikrecht, das für Militärangehörige, Polizei, Gerichtspolizei und Zollbeamte eingeschränkt ist. ${ }^{61}$

\section{c) Auflösung des Dienstvertrags}

Der Dienstvertrag eines Beamten kann nicht nur modifiziert, sondern auch einseitig vom Dienstherrn vollständig oder teilweise aufgehoben werden. Sind Rechtsvorschriften zum Nachteil des öffentlichen Interesses verletzt worden, muß eine Sanktion zur Wiedergutmachung festgesetzt werden. ${ }^{62}$

Die Auflösung eines Dienstverhältnisses erfolgt unter Anwendung der allgemeinen Regelungen des Arbeitsrechts. Das ZJU regelt zwei zusätzliche Gründe für eine ex legeAuflösung eines Dienstvertrags, nämlich, wenn der Beamte eine dienstliche Fortbildung nicht besteht oder zu einer Freiheitsstrafe von mehr als sechs Monaten (ohne Bewährung) verurteilt wird. ${ }^{63}$

57 Dieses Argument wurde auch als Begründung für die Gesetzesänderung angeführt. Poročevalec DZ, No. 57/05.

58 So z.B. für Soldaten (Art. 96 einschl. ZObr und Art. 53 einschl. ZSSloV).

59 Končar, in: Pavčnik/Polajnar-Pavčnik/Wedam-Lukić (Hrsg.), Temeljne pravice (Fußn. 3), S. 277.

60 Beispielsweise können in Tarifverträgen die Höhe einzelner Gehaltsstufen, Prämienzahlungen für eine zusätzliche Altersversicherung anstelle einer Valorisierung, die Gesamtsumme aller Leistungsboni im öffentlichen Dienst (unter Berücksichtigung bestimmter gesetzlicher Obergrenzen) sowie weitere Zusatzleistungen geregelt werden (siehe dazu die entsprechenden Art. des ZSPJS).

61 Art. 99 ZObr, 84 ZPol, 217 ZIKS-1 und 56 ZCS-1.

62 Virant, Delovna razmerja v javni upravi (Arbeitsverhältnisse in der Öffentlichen Verwaltung), Podjetje in delo, št. 6-7/2003, S. 1392, 1396.

Beispielsweise wird eine solche „Sanktion“ in Fällen vorgesehen, in denen ein Dienstvertrag trotz mangelnder Qualifikation des Betroffenen für die zu besetzende Stelle abgeschlossen oder eine erforderliche öffentliche Ausschreibung nicht durchgeführt wurde (Art. 74-77a ZJU).

63 Diese Bedingungen sind beispielsweise für Soldaten, die Polizei und Zollbeamte strenger (hier genügen kürzere Freiheitsstrafen für die Auflösung des Arbeitsvertrages). Blaha (Fußn. 48), Delavci in delodajalci, No. 2-3/2009, S. 359, 378. 
Darüber hinaus finden besondere Regelungen bei Beendigung des Dienstverhältnisses aus betrieblichen Gründen oder aus Gründen der Inkompetenz des Beamten Anwendung. ${ }^{64}$ Betriebliche Gründe im privaten Sektor können wirtschaftlicher, organisatorischer, technischer, strukturbedingter oder ähnlicher Art sein. Im öffentlichen Dienst dagegen zählen der Abbau oder die Privatisierung bestimmter öffentlicher Aufgaben, oder organisatorische, strukturelle, finanztechnische oder ähnliche Gegebenheiten zu den betrieblichen Beendigungsgründen, wie auch die Auflösung einer Verwaltungsstelle. Werden öffentliche Aufgaben auf eine andere Verwaltungsstelle, Kommune oder eine andere juristische Person des öffentlichen oder bürgerlichen Rechts übertragen, dann müssen auch alle Beamten übernommen werden. Die Regelung ist der Regelung des Betriebsübergangs im privaten Sektor ziemlich ähnlich, wenn auch die Richtlinie 2001/23/EG bei der Übertragung von Aufgaben im Zuge einer Umstrukturierung von Verwaltungsbehörden oder bei der Übertragung von Verwaltungsaufgaben von einer Behörde auf eine andere Behörde keine Anwendung findet. ${ }^{65}$

Ein Beamter ist als inkompetent für den öffentlichen Dienst anzusehen, wenn er den fachlichen Anforderungen des innegehabten Amtes nicht gerecht wird und die entsprechenden Leistungen nicht erbringt. ${ }^{66}$ In diesen Fällen ist der Beamte entsprechend der diesbezüglichen Regelungen des $Z J U$ an eine andere Dienststelle zu versetzen. ${ }^{67}$

Im Jahre 2002 wurde eine Regelung in das $Z J U$ aufgenommen, wonach Beamte bei Erreichen des vollen Pensionsalters in den Ruhestand versetzt wurden, sofern sie die für einen ungekürzten Bezug einer Alterspension erforderliche Dienstzeit abgeleistet hatten (,volle Pensionszeit"). Während in der gesetzlichen Rentenversicherung der Begriff „,volles Renteneintrittsalter" eindeutig geregelt ist, wird die für den Bezug einer (ungekürzten) Rente erforderliche Versicherungs- bzw. Beschäftigungszeit („,volle Pensionszeit“) nicht näher umschrieben. Den Begriff der ,vollen Pensionszeit“" verwendete das vormalige, bis Ende 1999 gültige Renten- und Invaliditätsversicherungsgesetz (ZIPZ). Das neue Renten- und Invaliditätsversicherungsgesetz (ZPIZ-1) fördert dagegen den längeren Verbleib im Arbeitsmarkt, um höhere Rentenanwartschaften erwerben zu können (und damit eine kürzere Inanspruchnahme).

Das Verfassungsgericht hob die zitierte Bestimmung des ZJU mit der Begründung auf, $68 \mathrm{da} ß$ das verfassungsrechtlich verankerte Rechtsstaatsprinzip eine hinreichend bestimmte und eindeutige Rechtsvorschrift erfordere. Der Inhalt und Zweck einer

64 Bei schuldhaftem Verhalten des Beamten finden die allgemeinen Regelungen des Arbeitsrechts Anwendung. Ebda., S. 374.

65 Art. 1(c) der Richtlinie 2001/23/EG des Rates vom 12.03.2001 zur Angleichung der Rechtsvorschriften der Mitgliedstaaten über die Wahrung von Ansprüchen der Arbeitnehmer beim Übergang von Unternehmen, Betrieben oder Unternehmens- oder Betriebsteilen, ABl. L 82 vom 22.03.2001, S. 16.

66 Die Arbeit bestimmter Beamter (z.B. von Soldaten) wird regelmäßig einer Beurteilung unterzogen. Fällt die Beurteilung negativ aus, kann der Arbeitsvertrag aufgelöst werden (Art. 94 ZObr).

67 Virant (Fußn. 62), Podjetje in delo, št. 6-7/2003, S. 1392, 1398.

68 Entscheidung No. U-I-227/06 vom 16.11.2006, OdIUS XV, 81. 
Rechtsvorschrift müsse - nötigenfalls durch Auslegung - erfaßbar sein. Da die Vorschrift des ZJU gegen das Gebot der Rechtssicherheit verstoße, verstoße sie auch gegen das Rechtsstaatsprinzip. Das Verfassungsgericht sah keine Notwendigkeit, die Vereinbarkeit der Vorschrift des ZJU mit dem verfassungsrechtlichen Gleichheitssatz zu prüfen. Allerdings verwies es auf eine seiner früheren Entscheidungen, die allgemeine arbeitsrechtliche Bestimmungen über die Zwangsverrentung außer Kraft setzte, ${ }^{69}$ und auf weitere Entscheidungen des Europäischen Gerichtshofs. ${ }^{70}$ Der Gesetzgeber kann Regelungen dahingehend treffen, daß der Dienstvertrag bei Erreichen eines bestimmten Alters automatisch aufgelöst wird. Voraussetzung ist aber, daß eine solche Regelung sachlich gerechtfertigt und zur Erreichung des verfolgten Gesetzeszwecks verhältnismäßig ist. 71

Für bestimmte Gruppen von Beamten ist noch immer eine Altersgrenze vorgeschrieben. Für Militärangehörige ist beispielsweise die ex lege-Auflösung des Dienstverhältnisses für das Ende des Jahres festgesetzt, in dem sie die Anspruchsvoraussetzungen für das Altersruhegeld erreichen. Für diesen Fall stellte das Verfassungsgericht keinen Verstoß gegen verfassungsrechtliche Grundsätze fest. ${ }^{72}$ Eine Altersgrenze ist auch für manche Träger öffentlicher Befugnisse vorgeschrieben. ${ }^{73}$

\section{d) Rechtsweg}

Das Recht auf gerichtlichen Schutz ist in der slowenischen Verfassung in Art. 23 als Menschenrecht für alle, einschließlich Beamte, verankert. Entscheidungen über die Rechte und Pflichten von Beamten sowie über alle gegen sie vorgebrachten Beschuldigungen müssen ohne Verzögerung vor einem unabhängigen, unparteiischen Gericht

69 Das Verfassungsgericht führte in der Entscheidung U-I-49/98 (25.11.1999, OdlUS VIII, 266) aus, daß das Rentenalter für Männer und Frauen in der gesetzlichen Rentenversicherung unterschiedlich sei. Damit werde bei der Beendigung des Beschäftigungsverhältnisses das Geschlecht des Arbeitnehmers und indirekt sein Alter berücksichtigt. Individuelle Merkmale wie Geschlecht und Alter erhalten im Arbeitsrecht und in der Rentenversicherung unverkennbar Gewicht. Während die Möglichkeit, in jüngerem Alter und mit einer kürzeren Versicherungszeit in den Ruhestand zu gehen, in der Rentenversicherung als Vorteil gesehen werden könne, könne dies bei der Beendigung eines Beschäftigungsverhältnisses eine Diskriminierung zur Folge haben. Das neue Betriebsverfassungsgesetz $(Z D R)$ enthält keine Vorschriften über die ex lege-Auflösung eines Beschäftigungsverhältnisses, wenn die Anspruchsvoraussetzungen für die Altersrente erfüllt sind.

70 Vgl. EuGH, Rs. C-262/84, Slg. 1986, 773 - Vera Mia Beets-Proper; EuGH, Rs. C-152/84, Slg. 1986, 723 - Marshall; EuGH, Rs. C-151/84, Slg. 1986, 703 - Roberts.

71 Vgl. die neuesten EuGH-Entscheidungen, wie z.B. Palacios de la Villa (Rs. C-411/05, Slg. 2007-I, 8531), Age Concern England (Rs. C-388/07, 5.3.2009).

72 Aufgrund der besonderen Anforderungen an den Dienst in der Armee soll es dem Gesetzgeber möglich sein, Dienstverhältnisse anders als in anderen Beschäftigungsbereichen zu regeln. Das Verfassungsgericht stellte keinen Verstoß gegen den verfassungsrechtlichen Gleichheitssatz fest (U-I329/04, 12.5.2005, OdIUS XIV, 27).

73 So werden Richter zum Ende des Kalenderjahres, in dem sie 70 Jahre alt werden, in den Ruhestand versetzt. 
verhandelt werden. Für die Rechtsweggarantie ist es unerheblich, ob die Zuständigkeit eines Verwaltungs-, Arbeits- oder Zivilgerichts oder eines anderen Gerichts eröffnet ist. Ist die gerichtliche Zuständigkeit jedoch unklar, dann könnte ein Verstoß gegen andere Grundrechte, wie beispielsweise das Recht auf Gleichheit vor dem Gesetz oder den gleichen Schutz der Rechte, vorliegen. ${ }^{74}$

Die Zuständigkeit der Verwaltungsgerichte ist eröffnet, wenn die Rechtmäßigkeit einer Verwaltungsentscheidung in Streit steht (beispielweise die Auswahl des besten Bewerbers im Zuge einer öffentlichen Ausschreibung oder die Ernennung in oder die Entlassung aus einem Amt oder einer Dienststelle). Demgegenüber ist die Zuständigkeit der Arbeitsgerichte eröffnet, wenn über Rechte und Pflichten aus einem Dienstverhältnis gestritten wird. In beiden Fällen ist eine vorherige Entscheidung der Beschwerdekommission vorzulegen. ${ }^{75} \mathrm{Ob}$ die Verwaltungs- oder Arbeitsgerichte bei Streitigkeiten über einseitige Verwaltungsentscheidungen, beispielsweise bei der Modifikation oder sogar der Beendigung von Dienstverhältnissen in bestimmten Fällen, zuständig sind, ist nicht geklärt. Dem ZJU kann keine Zuständigkeitsregelung entnommen werden, weil lediglich ein „Rechtsweg“ vorgeschrieben wird. Vertretbar ist es, die Zuständigkeit der Arbeitsgerichte anzunehmen. ${ }^{76}$

\section{Die Altersversorgung von Beamten}

\section{Das Verfassungsrecht}

Die slowenische Verfassung ist eine moderne Verfassung, die soziale Grundsätze und soziale Grundrechte normiert. Slowenien ist ein Sozialstaat (Art. 2), der seinen Bürgern das Recht auf soziale Sicherheit gewährt, einschließlich des Rechts auf eine Rente (Art. 50). Das slowenische Verfassungsgericht hat in seiner Rechtsprechung das Sozialstaatsprinzip, anders als das Rechtsstaatsprinzip, nicht stetig weiterentwickelt. Eine Ausnahme stellt der Grundsatz der Solidarität dar.

Alle Bürger, Staatsbedienstete eingeschlossen, haben ein Recht auf soziale Sicherheit. 77 Das Recht auf eine gesetzliche Rente wird gesondert erwähnt, was gleichzeitig die Frage aufwirft, welche Bedeutung dem beizumessen ist. ${ }^{78}$ Hintergrund ist die $\mathrm{Ge}$ währleistung eines angemessenen, auf geleistete Arbeit beruhenden Altersruhegelds aus

74 Blaha (Fußn. 48), Delavci in delodajalci, No. 2-3/2009, S. 359, 360.

75 Art. 24 ZJU.

76 Blaha (Fußn. 48), Delavci in delodajalci, No. 2-3/2009, S. 359, 371.

77 Die Verfassung gibt nur Mindestgarantien. Im internationalen Recht hat jeder als Mitglied der Gesellschaft ein Recht auf soziale Sicherheit.

78 Bubnov-Škoberne, Ustavna pravica do pokojnine? (Verfassungsrecht auf die Rente?), Izobraževanje in usposabljanje v javni upravi, IJU, No. 3/2002, S. 235, 243. 
der Rentenversicherung. Die gesetzliche Alterssicherung ist einer und zugleich der wichtigste der verfassungsrechtlich, zur Durchsetzung des Rechts auf soziale Sicherheit vorgesehenen Sozialversicherungszweige (Art. 50 Abs. 2). Die inhaltliche Ausgestaltung und die Regelung der Anspruchsvoraussetzungen obliegen dem Gesetzgeber und sind in der Verfassung nicht vorgegeben. Dadurch, daß das Recht auf eine Altersrente in der Verfassung explizit genannt ist, hat das Verfassungsgericht einen zusätzlichen Prüfungsrahmen, um die Verfassungsmäßigkeit der Eingriffe des Gesetzgebers im Bereich der Alterssicherung zu beurteilen. 79

Die slowenische Verfassung schreibt dem Gesetzgeber nicht vor, ein besonderes Altersvorsorgesystem für Beamte einzurichten. Unter Beachtung des Sozialstaatsprinzips hat der Gesetzgeber bei der Gewährleistung und Ausgestaltung des Rechts auf soziale Sicherheit einen großen Ermessensspielraum. Bei der Nennung der Rentenversicherung verwendet die Verfassung aber den Singular, d.h. es muß eine (Renten-)Versicherung eingerichtet werden. Die Verfassung schreibt die Einrichtung einer separaten, nicht beitragspflichtigen Altersversorgung für Beamte somit nicht vor.

\section{Die verschiedenen Altersvorsorgesysteme für Beamte}

Träger öffentlicher Befugnisse und Beamte, deren Dienstverhältnis auf einem Dienstvertrag beruht, sind in der allgemeinen gesetzlichen Renten- (und Invaliditäts-) Versicherung versichert. Für bestimmte Gruppen von Beamten gelten jedoch Sonderbestimmungen. Zudem ist die allgemeine Rentenversicherung oder erste Säule des Alterssicherungssystems (Regelsicherungssystem) für bestimmte Beschäftigungsverhältnisse, einschließlich solcher im öffentlichen Dienst, als obligatorische Alterszusatzversicherung vorgesehen.

Die zweite Säule besteht aus der freiwilligen, ergänzenden Alterszusatzversicherung, die vom Staat geregelt und gefördert, aber von privaten Versicherungsfonds verwaltet wird. Auch Beamte können ergänzende Alterszusatzversicherungen abschließen. Zudem gibt es ein besonderes System für eine kollektive Alterszusatzversicherung für Beamte.

Die dritte Säule bilden private (individuell ausgerichtete) Rentenversicherungen, die auf eigenen Rentensparkonten basieren und nur von privaten Versicherungsgesellschaften angeboten werden. Das Zivil- (bzw. Versicherungs-)Recht findet Anwendung, eine steuerrechtliche Sonderbehandlung erfolgt nicht. Die dritte Säule des Alterssicherungssystems ist nicht Gegenstand nachfolgender Ausführungen.

79 Nach der Rechtsprechung des Verfassungsgerichts ist das Recht jedes Einzelnen, unter angemessenen Rahmenbedingungen Rentenanwartschaften zu erwerben und eine beitragsfinanzierte Rente zu beziehen, das Kernelement des Rentenrechts, das die soziale Sicherheit gewährleistet (Up-360/05, 2.10.2008, Amtsblatt RS, No. 118/08). Die verfassungsrechtliche Rentengarantie bedeutet nicht, dem Einzelnen einzig ein Existenzminimum zu bieten. 
Erfüllt ein Versicherter, einschließlich Beamter, die Anspruchsvoraussetzungen für den Bezug einer Altersrente aus der ersten (und möglicherweise auch der zweiten) Säule nicht, kann er unter Umständen Anspruch auf eine staatliche Rente haben, die als eine Art und auf diesen Personenkreis zugeschnittene Sozialhilfe zu qualifizieren ist.

Werden die Bezugsvoraussetzungen einer Rente nicht erfüllt oder liegt das Einkommen trotz bestehender Rentenansprüche unter einer bestimmten Einkommensgrenze, dann kann allgemeine Sozialhilfe beantragt werden.

\section{a) Die gesetzliche Rentenversicherung}

\section{aa) Allgemeiner Geltungsbereich}

Grundsätzlich ist die gesamte erwerbstätige Bevölkerung 80 in der gesetzlichen Rentenversicherung pflichtversichert, die in dem Renten- und Invaliditätsversicherungsgesetz geregelt ist (Zakon o pokojninskem in invalidskem zavarovanju-ZPIZ-1). ${ }^{81}$

Das ZPIZ-1 schließt ausdrücklich alle für den Staat und die Kommunen tätigen Träger öffentlicher Befugnisse in die allgemeine Rentenversicherung ein. ${ }^{82}$ Andererseits verweisen alle Gesetze, die Regelungen für den öffentlichen Dienst enthalten, auf die allgemeine Rentenversicherung. Eine Besonderheit ist, daß für diesen Personenkreis nach Auslaufen der Amtszeit ein Anspruch auf eine Abfindung vorgesehen ist, wenn sie aus sachgerechten Gründen nicht an ihrem alten Arbeitsplatz beschäftigt werden, keine neue Beschäftigung finden können oder in den Ruhestand gehen. In einem solchen Fall gründet die Beitragszahlung nicht auf der ausgeübten beruflichen Tätigkeit, sondern auf der (beendeten) Ausübung eines bestimmten Amtes und den damit verbunden Besonderheiten ${ }^{83}$. Das ZPIZ-1 umfaßt alle in Slowenien beschäftigten Arbeitnehmer, die den Großteil der Versicherten ausmachen. ${ }^{84}$ Das Gesetz schließt die Beamten mit ein, auch wenn sie nicht explizit Erwähnung finden.

80 D.h. Arbeitnehmer, Beamte, Selbständige und Landwirte (wobei der erwirtschaftete Gewinn von Selbständigen und Landwirten wenigstens die Höhe des Mindestlohns erreichen muß).

81 Amtsblatt RS, No. 106/1999, zuletzt geändert 2006. Es löste das Renten- und Invaliditätsversicherungsgesetz (ZPIZ) von 1992 ab.

82 Pflichtversichert sind gewählte und ernannte Träger öffentlicher oder anderer Ämter, die in Organen der Legislative, Exekutive oder Judikative in der Republik Slowenien oder in Behörden der kommunalen Selbstverwaltung tätig sind, wenn sie für diese Amtstätigkeit ein Gehalt beziehen (Art. 13 ZPIZ-1).

83 Beispielsweise das Wahl- oder Ernennungsverfahren, befristete Mandate, Haftung, Erschwernis, Unvereinbarkeit mit anderen Tätigkeiten, Wahrung der Unparteilichkeit in besonders schwierigen Situationen, etc., vgl. Strban, Pravni vidiki socialne varnosti za javne uslužbence (Rechtliche Aspekte der soziale Sicherheit für Beamten), Pravni in drugi vidiki uslužbenskih razmerij, SECLI, 2001, S. $1,7$.

84 Die Arbeitnehmer machen etwas mehr als $85 \%$ aller Versicherten aus (www.zpiz.si). 
Im Übrigen enthält das ZJU keine besonderen Vorschriften über eine Sozial- (und damit auch Alters-)Versicherung für Beamte. Deshalb müssen die allgemeinen Regeln der gesetzlichen Rentenversicherung angewendet werden.

\section{bb) Leistungen}

$\mathrm{Zu}$ den Hauptleistungen der gesetzlichen Rentenversicherung gehört die Altersrente (starostna pokojnina). Die Voraussetzungen für einen Mindestanspruch hängen von der Versicherungs- bzw. Beschäftigungszeit und dem Alter des Versicherten ab. Das Renteneintrittsalter variiert je nach Versicherungs- bzw. Beschäftigungszeit.

Eine Altersrente kann bezogen werden, wenn

- das Alter von 58 Jahren und eine Versicherungszeit von 40 Jahren für Männer bzw. 38 Jahren für Frauen erreicht sind, 85

- das Alter von 63 Jahren für Männer bzw. 61 Jahren für Frauen und eine Versicherungszeit von 20 Jahren erreicht sind,

- das Alter von 64 Jahren für Männer bzw. 63 Jahren für Frauen und eine Versicherungszeit von mindesten 15 Jahren erreicht sind. ${ }^{86}$

Das Mindesteintrittsalter für den Bezug einer Altersrente kann in zwei Fällen gesenkt werden:

- auf höchstens 58 Jahre für Männer bzw. 56 Jahre für Frauen als ein Elternteil und für jedes Kind, das dieser mindestens fünf Jahre lang erzogen hat,

- nicht unter 55 Jahre für Frauen, die vor der Volljährigkeit erwerbstätig waren, d.h. zwischen 15 Jahren als dem Mindestalter für den Abschluß eines Beschäftigungsvertrags und 18 Jahren.

Die Höhe der Altersrente zu bestimmen, bereitet keine Schwierigkeiten. Die Altersrente errechnet sich als ein Prozentsatz aus dem Rentensockel. Der Rentensockel wird aus dem Durchschnittslohn der besten 18 ohne Unterbrechung zurückgelegten Versicherungsjahre eines Versicherten gebildet. Das Jahr, in dem der Versicherte in den Ruhestand geht, zählt nicht. Die Höhe der Altersrente ist nur mit dem limitierten Rentensockel beschränkt. Der Prozentsatz kann mit weiterer Versicherung unbegrenzt steigen. ${ }^{87}$

Aufgrund der unterschiedlichen Anspruchsvoraussetzungen für Männer und Frauen, ist auch der Prozentsatz, der für die exakte Berechnung der Rentenhöhe zugrunde gelegt

85 Zusätzliche Zeiten wie Studienzeiten, Wehrdienst, Arbeitslosigkeit sowie Zeiten aus einer obligatorischen Alterszusatzversicherung werden bei der Berechnung der Altersrente berücksichtigt, wenn Beiträge entrichtet wurden, vgl. Art. 42, 192 und 193 ZPIZ-1.

86 Die Übergangsphase bis 2014 findet keine Berücksichtigung. Vgl. Cvetko, in: Belopavlovič u.a., Zakon o pokojninskem in invalidskem zavarovanju s komentarjem (Gesetz über die Renten- und Invaliditätsversicherung mit Kommentar), 2000, S. 213.

872009 beträgt der Mindest-Rentensockel 535,51 Euro, der maximale Rentensockel 2142,04 Euro (www.zpiz.si). 
wird, unterschiedlich. ${ }^{88}$ Für eine Versicherungszeit von 15 Jahren (die für einen Mindestanspruch auf Altersrente erforderlich ist) erhalten Männer $35 \%$ und Frauen 38 \% des Rentensockels. Der Prozentsatz erhöht sich mit jedem weiteren Versicherungsjahr um 1,5 Prozentpunkte. So erhalten ein Mann nach vollständigem Ablauf von 40 Erwerbsjahren und eine Frau nach vollständigem Ablauf von 38 Erwerbsjahren eine Altersrente von $72,5 \%$ des Rentensockels. Nach diesen Berechnungsregeln kann eine (Mindest-)Rente nicht unter $35 \%$ des Mindestsockels fallen. 89

Eine Besonderheit stellt das sogenannte Bonus-Malus-System dar. Bei diesem System erhält ein Versicherter einen zusätzlichen Prozentpunkt, wenn er nach Erfüllung der normierten Versicherungszeit oder nach Erreichen des Renteneintrittsalters (im Regelfall 63 Jahre für Männer, 61 Jahre für Frauen) weiterhin versichert bleibt. Andererseits erhalten Versicherte Abzüge, wenn sie das Altersruhegeld vor Erreichen des Renteneintrittsalters in Anspruch nehmen. Die vorgenommenen Erhöhungen und Senkungen sind dauerhaft. D.h., eine Altersrente wird beispielsweise zum Zeitpunkt des Erreichens des vollen Renteneintrittsalters nicht neu berechnet. Kritik am Bonus-Malus-System wird dahingehend geübt, daß es nicht nur nach dem Alter der Versicherten unterscheidet, sondern auch nach Einkommen und Vermögen. Im Nachteil befinden sich Versicherte mit geringerem Einkommen und Vermögen, die, um eine angemessene Rente zu erhalten, länger arbeiten müssen (weil sie sich einen Malus nicht leisten können und gerade$\mathrm{zu}$ auf einen Bonus angewiesen sein könnten). Das gilt natürlich nur für den Fall, daß sie zur Erwerbstätigkeit in der Lage sind. ${ }^{90}$

Das ZPIZ-1 handhabt die Anspruchsvoraussetzungen für den Bezug einer Altersrente, die Sondergesetze regeln können, weniger streng. Allerdings normiert das ZPIZ-I bestimmte Grenzen ${ }^{91}$ und stellt sicher, daß der aufgrund der allgemeinen Regelungen und der Sondervorschriften entstehende Differenzbetrag bzw. die gesamte Altersrente (wenn kein Rentenanspruch nach den allgemeinen Regelungen besteht) von der Staatskasse getragen wird.

Für den öffentlichen Dienst bestanden Sonderregelungen. Zum Beispiel konnten Parlamentsabgeordnete am Ende ihrer Mandatszeit und einer Dienstzeit von 25 Jahren ohne Rücksicht auf ihr Alter einen Rentenanspruch geltend machen. Außerdem wurde eine

88 Wenn die unterschiedliche Berechnung notwendigerweise und sachlich korrekt an das unterschiedliche Renteneintrittsalter gebunden ist, kann sie als Ausnahme vom Gleichheitsgrundsatz von Männern und Frauen in der EG gelten. In diesem Zusammenhang siehe beispielsweise die EuGHEntscheidung De Vriendt und andere Fälle (C-377/96-384/96, Slg. 1998, I-2105).

89 2009: 187,43 Euro (www.zpiz.si).

90 Strban, Nekateri primeri (ne)utemeljenega razlikovanja v pravu socialne varnosti Slovenije in ES (Einige Beispiele der (un)begründete Unterscheidung im slowenischen und EG Recht), Delavci in delodajalci, 2007 (Sonderausgabe), S. 67, 86.

91 Das Renteneintrittsalter soll für Männer auf keinen Fall unter 55 Jahre sinken und die Versicherungszeit nicht weniger als 35 Jahre betragen. Für Frauen sollte das Renteneintrittsalter nicht unter 53 Jahre sinken und die Versicherungszeit nicht weniger als 33 Jahre betragen. Vgl. Art. 155 ZPIZ1. 
günstigere Berechnung des Ruhegehalts vorgesehen. ${ }^{92}$ Das Verfassungsgericht hob diese Sonderregelung auf. Es stellte fest, daß es nicht per se verfassungswidrig sei, wenn Parlamentsabgeordneten günstigere Pensionsansprüche zugesichert werden, solange kein Widerspruch zu rechts- und sozialstaatlichen Grundsätzen vorliege. Folglich sollten Sonderbestimmungen nicht (willkürlich) einzig auf die Amtszeit abstellen, sondern die besondere Art und Dauer des Amtes berücksichtigen. Das angemessene Verhältnis zwischen diesen Erwägungen und den Rechten, die anderen Bürgern zustehen, sollte gewahrt bleiben. Nur insoweit dürfen Sonderbestimmungen von den allgemeinen Regelungen der Rentenversicherung unter Berücksichtigung der allgemeinen sozialen Verhältnisse (und des Gleichheitsprinzips) abweichen. ${ }^{93}$

Sonderregelungen gibt es außerdem für bestimmte Gruppen von Beamten, beispielsweise für den Polizeidienst (Polizeibeamte und sonstige bei der Polizei beschäftigte Personen). Das Renteneintrittsalter liegt für Männer bei 45 Jahren und für Frauen bei 40 Jahren. Darüber hinaus müssen Männer eine Beschäftigungszeit von mindesten 30 Jahren und Frauen von mindestens 25 Jahren nachweisen, wobei 15 Jahre auf die Tätigkeit im Polizeidienst entfallen müssen. Für den Polizeidienst sind nicht nur die Bezugsvoraussetzungen für eine Altersrente, sondern ist auch die Rentenberechnung günstig ausgestaltet. ${ }^{94}$

Für manche Staatsbedienstete, wie Richter oder Militärangehörige, bestand in den 1990er Jahren, als das Justiz- und Militärwesen reformiert wurde, die zeitlich befristete Möglichkeit, Altersrente unter günstigeren Voraussetzungen zu beantragen. Arbeitnehmer und Staatsbedienstete haben Anspruch auf eine Teilrente (delna pokojnina). Voraussetzung ist, daß der Versicherte die Anspruchsvoraussetzungen für die Altersrente erfüllt. Bei Bezug einer Teilrente wird das Beschäftigungsverhältnis teilweise aufrecht erhalten, jedoch nur bis zur Hälfte der vollen Arbeitszeit, und der Versicherte erhält die Hälfte der ihm zustehenden Altersrente.

Arbeitnehmer und Staatsbedienstete können zusätzliche Leistungen aus der gesetzlichen Rentenversicherung beziehen. Hierzu gehört die Zulage für Rentner mit sehr niedrigen Altersrenten. Als Leistung der Sozialhilfe unterliegt diese Zulage einer Bedürfnisprüfung und wird seit 2008 in einem separaten Gesetz ${ }^{95}$ geregelt. Da in Slowenien noch keine gesetzliche Pflegeversicherung errichtet wurde, kann bei der gesetzlichen Renten-

92 Die Höhe der Rente sollte bei einer 25jährigen Dienstzeit $70 \%$ des Rentensockels betragen. Der Prozentsatz sollte für jedes über diesen 25 Dienstjahren liegende Jahr um zwei Prozentpunkte bis zur Grenze von 85 \% des Rentensockels angehoben werden (Art. 39 ZPos von 1992).

93 Entscheidung No. U-I-135/92, 30.6.1994, OdIUS III, 84. Strban, Pravica do pokojnine pod ugodnejšimi pogoji (Anspruch auf die Rente unter günstigere Bedingungen), Podjetje in delo, No. 2/1999, S. 328, 331.

94 Die Altersrente für Beschäftigte bei der Polizei beträgt $65 \%$ des Rentensockels. Für jedes Beschäftigungsjahr, das bei Männern über 30 und bei Frauen über 25 Beschäftigungsjahre hinausgeht, wird der Prozentsatz um zwei Prozentpunkte bis zur Grenze von 85 \% des Rentensockels angehoben, vgl. Art. 87 ZPol.

95 Zakon o varstvenem dodatku - ZvarDod, Amtsblatt RS, No. 10/08. 
versicherung ein Anspruch auf Pflegegeld geltend gemacht werden, wenn ein Rentenempfänger die gewöhnlichen Verrichtungen des täglichen Lebens teilweise oder gar nicht mehr bewältigen kann. Alle Rentenempfänger haben außerdem einen Anspruch auf eine jährliche Zulage.

\section{cc) Finanzierung und Verwaltung}

Die slowenische gesetzliche Rentenversicherung ist als ein leistungsdefiniertes System zu qualifizieren, das im Umlageverfahren durch Beiträge der Versicherten finanziert wird. Beitragsschuldner sind die Versicherten (Staatsbedienstete eingeschlossen) und ihre Arbeitgeber bzw. Dienstherrn. Leistungen, die als eine Art Sozialhilfe zu qualifizieren sind, werden aus der Staatskasse finanziert. Der Staat ist zudem verpflichtet, mögliche Verluste des Versicherungsträgers auszugleichen.

Die Verwaltung der gesetzlichen Rentenversicherung (und ihres Fonds) ist dem eigenständigen Renten- und Invaliditätsversicherungsinstitut der Republik Slowenien übertragen (Zavod za pokojninsko in invalidsko zavarovanje Slovenije). Dieses Institut wurde vom Staat errichtet und unterliegt dem öffentlichen Recht. Die in ihrer Funktion dezentrale Einrichtung hat eine eigene Rechtspersönlichkeit und gehört nicht der staatlichen Verwaltung an. Wie der Zusammensetzung der Geschäftsführung zu entnehmen ist, ${ }^{96}$ ist sie mit Selbstverwaltungskompetenz ausgestaltet und mit Regional- und Zweigstellen zentralistisch strukturiert.

\section{b) Die obligatorische Alterszusatzversicherung}

In der obligatorischen Alterszusatzversicherung sind die Versicherten der gesetzlichen Rentenversicherung versichert, die schwere, gesundheitsschädigende Tätigkeiten ausüben oder solche Arbeiten verrichten, die nur bis zu einem bestimmten Alter geleistet werden können. Eine entsprechende Liste wird mit Einverständnis der Arbeitnehmergewerkschaften und der Arbeitgeberverbände vom Arbeitsministerium herausgegeben. Darunter sind auch Arbeitsstellen, auf denen Beamte, wie beispielsweise Polizeiund Zollbeamte oder Soldaten, beschäftigt werden. ${ }^{97}$

Ein Versicherter hat Anspruch auf eine monatliche Berufsrente (poklicna pokojnina)

- bis die Bezugsvoraussetzungen einer allgemeinen Altersrente erfüllt sind,

- als gekürzte Berufsrente neben der allgemeinen Altersrente.

Nach dem Rentenplan der obligatorischen Alterszusatzversicherung muß eine Versicherungszeit von 40 Jahren (Männer) bzw. von 38 Jahren (Frauen) nachgewiesen werden. Beschäftigungszeiten, die in den oben zitierten Arbeitsstellen zurückgelegt werden,

96 Vertreter der Arbeitgeber- und Arbeitnehmerorganisationen (Gewerkschaften), Leistungsempfänger (Empfänger von Alters- und Invaliditätsrenten) und Regierungsvertreter, vgl. Art. 266 ZPIZ-1.

97 Im Gesetz ist geregelt, für welche Aufgabenbereiche im Militärdienst eine obligatorische Rentenzusatzversicherung vorgeschrieben ist (Art. $60 \mathrm{ZSSloV}$ ). 
werden fiktiv auf die Versicherungszeit zugerechnet. ${ }^{98}$ Zudem werden diese Arbeitsstellen in fünf verschiedene Gruppen unterteilt, wobei für jede Gruppe ein eigenes Mindestalter für den Bezug der Berufsrente vorgeschrieben ist. 99

Die obligatorische Alterszusatzversicherung wird ausschließlich durch Arbeitgeberbeiträge finanziert, die je nach Art der Arbeitsstätte unterschiedlich hoch sind. ${ }^{100}$ Das System ist kapitalgedeckt, die Beiträge werden auf individuelle Konten der Versicherten eingezahlt. Folglich hängt die Höhe der Berufsrente von der Höhe der eingezahlten Beiträge und der erfolgreichen Verwaltung der Versicherungsträger ab. Der festgesetzte Mindestertrag dient der Ermittlung einer Mindestrente. 101 Das System kann als beitragsdefiniertes System mit leistungsdefinierten Komponenten bezeichnet werden.

Versicherungsträger ist ein besonderer, geschlossener Rentenanlagefonds, nämlich der Fonds der obligatorischen Alterszusatzversicherung. Der Fonds steht im Eigentum der Versicherten und wird vom Kapitalfonds der Renten- und Invaliditätsversicherung verwaltet, einer vom Staat eingerichteten Aktiengesellschaft. Unter besonderen, gesetzlich normierten Bedingungen, können auch andere Rentenversicherungsgesellschaften als Träger obligatorischer Alterszusatzversicherungen fungieren, was bisher in der Praxis nicht vorgekommen ist.

\section{c) Die freiwillige Alterszusatzversicherung}

Die freiwillige Alterszusatzversicherung ist eine private Rentenversicherung (zweite Säule der Rentenversicherung), die von zertifizierten Versicherungsfonds angeboten wird und im ZPIZ-1 geregelt ist. Hauptmerkmal ist, daß Arbeitnehmer und Arbeitgeber besondere Steuervergünstigungen erhalten, wenn die Versicherungsprämie gemäß einem zertifizierten Rentenplan eingezahlt wird. Der Einrichtung einer freiwilligen ergänzenden Alterszusatzversicherung müssen der Arbeitgeber und die (meist durch eine Gewerkschaft vertretenen) Arbeitnehmer zustimmen.

In der staatlichen gesetzlichen Rentenversicherung versicherte Personen einschließlich Beamte können einer freiwilligen ergänzenden Alterszusatzversicherung beitreten.

Sie können sich

- selbst versichern (Individualversicherung) oder

- über ihren Arbeitgeber versichern, wenn er die Versicherungsprämie (teilweise oder vollständig) entrichtet (Kollektivversicherung). Wenn er nur einen Teil der

98 Ein Viertel der Versicherungszeit aus der obligatorischen Alterszusatzversicherung (d.h. drei Monate pro Jahr) wird zu der gesamten Versicherungszeit hinzugerechnet.

99 Beispielsweise sind für die Gruppe I als Rentenalter 55,5 Jahre (Männer) bzw. 52 Jahre (Frauen) sowie für die Gruppe V 47,5 Jahre (Männer) bzw. 44 Jahre (Frauen) vorgesehen (www.kapitalskadruzba.si).

100 Die Beitragshöhe liegt zwischen 4,2 \% und 12,6 \% (www.kapitalska-druzba.si).

101 Die Mindestertragsrate liegt bei $60 \%$ des durchschnittlichen Jahres-Zinssatzes bestimmter Staatsanleihen (www.kapitalska-druzba.si). 
Prämie finanziert, kann der Versicherte den Restbetrag bis zu einer gesetzlich festgelegten Obergrenze selbst zahlen.

Sowohl die Arbeitgeber- als auch die Arbeitnehmerprämie kann steuerlich abgesetzt werden. Wird eine höhere Prämie gezahlt, als gesetzlich festgelegt ist, werden für den überschießenden Betrag keine Steuervergünstigungen gewährt. Jeder Beschäftigte kann gleichzeitig in einer freiwilligen und einer kollektiven Versicherung versichert sein. Die gesetzlich festgelegte Obergrenze für Steuervergünstigungen gilt in diesem Fall jedoch für beide zusammen.

Aus der freiwilligen ergänzenden Alterszusatzversicherung ist der Anspruch auf eine ergänzende Altersrente (dodatna starostna pokojnina) von maßgeblicher Bedeutung. Voraussetzung ist ein Mindestalter von 58 Jahren, eine Versicherungszugehörigkeit in der freiwilligen ergänzenden Zusatzversicherung von mindestens zehn Jahren, sowie der Bezug einer Altersrente aus der gesetzlichen Rentenversicherung, für die das Renteneintrittsalter für Männer und Frauen unterschiedlich ist. Ein solch enger Bezug zur gesetzlichen Alterssicherung kann unter Umständen Probleme bereiten. Wenngleich eine Unterscheidung aufgrund des Geschlechts in der gesetzlichen Rentenversicherung zulässig ist, so ist sie doch in der ergänzenden Alterszusatzversicherung verboten. ${ }^{102}$

Das ZPIZ-1 sieht eine Möglichkeit auf eine vorzeitige Alterszusatzrente vor, wenn ein Beschäftigter nicht mehr in dem staatlichen System versichert ist, mindestens 15 Jahre einer freiwilligen ergänzenden Zusatzversicherung angehörte und das 53. Lebensjahr vollendet hat. Allerdings muß das Recht auf eine vorzeitige Alterszusatzrente im Rentenplan niedergelegt sein, was in der Praxis selten der Fall ist.

Träger sind zertifizierte Versicherungsfonds, d.h. ein offener oder geschlossener Rentenanlagefonds oder eine Rentenversicherungsgesellschaft. Der Versicherungsfonds hat keine eigene Rechtspersönlichkeit. Er bildet das Vermögen der versicherten Personen, das von einer Versicherungsgesellschaft, einer Bank oder einer Rentenversicherungsgesellschaft verwaltet wird. Der Versicherungsfonds muß eine Aktiengesellschaft mit einer bestimmten Zahl von Versicherten sein.

\section{d) Die kollektive Alterszusatzversicherung für Beamte}

Einige Jahre nach der Einführung des Drei-Säulen-Systems durch das ZPIZ- 1 entschied sich ein Großteil der Beamten, dem freiwilligen ergänzenden Alterszusatzsystem (zweite Säule) nicht beizutreten. Daraufhin beschlossen die Sozialpartner - in diesem Fall die Regierung und die Gewerkschaften des öffentlichen Dienstes - im Jahre 2003

102 So EuGH, Rs. C-262/88, Slg. 1990-I, 1889 - Barber; Richtlinie 2006/54/EG des Europäischen Parlaments und des Rates vom 5. Juli 2006 zur Verwirklichung des Grundsatzes der Chancengleichheit von Männern und Frauen in Arbeits- und Beschäftigungsfragen (Neufassung) ABl. L 2004 vom 26.07.2006, S. 23; Strban (Fußn. 90), Delavci in delodajalci, 2007 (Sonderausgabe), S. $67,76$. 
die (zuvor vereinbarte) Valorisierung der Gehälter dauerhaft in Prämien für eine kollektive ergänzende Alterszusatzversicherung für Beamte umzuwandeln. Ein Vertragsanhang zu den Tarifabkommen für den Öffentlichen Sektor erläutert dieses Sondersystem. ${ }^{103}$ Ende 2003 wurde daraufhin das Gesetz über die kollektive Alterszusatzversicherung für Beamte (Zakon o kolektivnem dodatnem pokojninskem zavarovanju za javne uslužbence - ZKDPZJU) ${ }^{104}$ verabschiedet und im Jahr 2004 ein separater Tarifvertrag geschlossen. ${ }^{105}$ Das ZKDPZJU ist lex specialis hinsichtlich der Bestimmungen über die freiwillige ergänzende Alterszusatzversicherung, die im ZPIZ-1 geregelt sind, wobei das ZPIZ-1 immer dann Anwendung findet, wenn das ZKDPUJU nicht greift.

Ziel des Sondersystems war nicht nur, Staatsbediensteten im Alter Einkommenssicherheit zu gewährleisten, sondern auch, die Drei-Säulen-Struktur der Alterssicherung durch die Beschäftigten im öffentlichen Dienst zu stärken. 106

Das Sondersystem richtet sich zwar an Beamte, schließt jedoch darüber hinaus die Beschäftigten des gesamten öffentlichen Dienstes mit ein. Ex lege umfaßt es die Staatsbediensteten, d.h. die Träger öffentlicher Befugnisse (wie Richter und Parlamentsabgeordnete) und Beamte sowie alle beim Staat, den Kommunen oder juristischen Personen des öffentlichen Rechts Beschäftigten. ${ }^{107}$ Grundsätzlich sind die Leistungen für Staatsbedienstete dieselben wie bereits oben erwähnt, d.h. ein zusätzliches Altersruhegeld und eine vorzeitige Alterszusatzrente. Eine Sonderregelung gibt es für ältere Versicherte, die in den Ruhestand gehen werden, bevor das System zehn Jahre besteht. 108

Weil diese Versicherung de facto ein Ersatz für eine Gehaltssteigerung ist, muß sie von Arbeitgebern des öffentlichen Sektors zwingend errichtet werden, die auch je nach der Länge der Dienstzugehörigkeit des jeweiligen Versicherten in 36 Kategorien eingeteilte Prämien zahlen. ${ }^{109}$ Die Prämien sind für die Beschäftigten des öffentlichen Dienstes (die auf einen Teil ihres Gehaltes verzichtet haben) steuerfrei. Der Versicherte kann die Prämie aber auch selbst einzahlen. In einem solchen Fall kann der gesamte

103 Kalčič, Kolektivno dodatno pokojninsko zavarovanje za javne uslužbence (Kollektive Zusätzliche Rentenversicherung für Beamten), Delavci in delodajalci, No. 2-3/2004, S. 457, 466, 473.

104 Amtsblatt RS, No. 126/03.

105 Tarifabkommen über die Einrichtung eines Alterssicherungsplans für Beamte (Kolektivna pogodba o oblikovanju pokojninskega načrta za javne uslužbence), Amtsblatt RS, No. 11/04.

$106 \mathrm{Zu}$ dieser Zeit traten ca. 156.000 Beamte der Alterszusatzversicherung bei, während ca. 210.500 Arbeitnehmer bereits freiwillig zusatzversichert waren. Kalčič (Fußn. 103), Delavci in delodajalci, No. 2-3/2004, S. 457, 474.

107 Pogačar, Dodatno pokojninsko zavarovanje: Kolektivno dodatno pokojninsko zavarovanje za javne uslužbence (Zusätzliche Rentenversicherung: Kollektive Zusätzliche Rentenversicherung für Beamten), Pravosodni bilten, No. 3/2008, S. 135, 138.

108 Ein Anspruch auf ein zusätzliches Altersruhegeld entsteht, sobald eine Altersrente aus der gesetzlichen Rentenversicherung bezogen wird, oder aber ein Anspruch auf Auszahlung eines bestimmten Betrages besteht. Vgl. Kalčič (Fußn. 103), Delavci in delodajalci, No. 2-3/2004, S. 457, 472.

109 Für alle Staatsbediensteten wird eine Grundprämie von 24,20 Euro gezahlt sowie Zusatzprämien, die bis zu 28,66 Euro betragen und in 36 Gruppen unterteilt sind (2009). In der obersten Gruppe beläuft sich die Prämie auf 52,86 Euro (www.kapitalska-druzba.si). 
Steuervorteil nach ZPIZ-1 geltend gemacht werden. ${ }^{110}$ Die Prämien werden in einen besonderen, geschlossenen Rentenanlagefonds für Beamte eingezahlt.

Die Verwaltung erfolgt durch den bereits erwähnten Kapitalfonds der Renten- und Invaliditätsversicherung. Wenn die Anspruchsvoraussetzungen erfüllt sind, kann der Versicherte entweder eine Versicherungsgesellschaft frei wählen, die die Zusatzrente auszahlt, oder er kann aus drei Angeboten auswählen, die der Kapitalfonds der Rentenund Invaliditätsversicherung zu offerieren hat. 111

\section{e) Die staatliche Rente}

Im Jahr 2000 wurde mit der Rentenreform die staatliche Rente (državna pokojnina) eingeführt. Obwohl diese Form der Rente im ZPIZ-1 geregelt ist, handelt es sich um keine Versicherungsleistung, sondern um Sozialhilfe, die einer Bedürfnisprüfung unterliegt und aus der Staatskasse finanziert wird. Anspruchsberechtigt sind nur Personen mit ständigem Wohnsitz in Slowenien, die keinen Anspruch auf eine Rente aus der gesetzlichen Sozialversicherung haben (weder in Slowenien noch in einem anderen Land) und deren Einkommen einen bestimmten Höchstbetrag nicht übersteigt. Voraussetzung ist ferner, daß der Antragsteller 65 Jahre alt und im Laufe seines aktiven Erwerbslebens 30 Jahre in Slowenien wohnhaft gewesen ist, d.h. zwischen dem vollendeten 15. und 65 . Lebensjahr. Die staatliche Rente wird für alle Leistungsberechtigten in gleicher Höhe bewilligt und beträgt ein Drittel des Mindestrentensockels. ${ }^{112}$

Darüber hinaus erhielten Rentner mit den niedrigsten Rentenbezügen 2008 eine einmalige, durch ein Sondergesetz geregelte Zulage. ${ }^{113}$ Wenn ein Betroffener die Anspruchsvoraussetzungen weder für eine Altersrente noch für die staatliche Rente erfüllt, kann er unter Umständen die allgemeine Sozialhilfe in Anspruch nehmen. Die im Sozialhilfegesetz normierten Voraussetzungen 114 müssen erfüllt sein.

\section{Abschließende Bemerkungen und die weitere Entwicklung de lege ferenda}

Die inhaltliche Ausgestaltung der Rechtsstellung von Beamten erfolgt sowohl durch arbeitsrechtliche als auch durch verwaltungsrechtliche Vorschriften. Die Verbindung dieser beiden Rechtsgebiete spiegelt sich im Recht der sozialen Sicherheit wider.

110 Kalčič (Fußn. 103), Delavci in delodajalci, No. 2-3/2004, S. 457, 471.

111 Pogačar (Fußn. 107), Pravosodni bilten, No. 3/2008, S. 135, 142.

1122009 beläuft sich die staatliche Rente auf 178.32 Euro (www.zpiz.si).

113 Zakon o enkratnem pokojninskem dodatku-ZEPokDod, Amtsblatt RS, No. 68/08.

114 Zakon o socialnem varstvu - ZSV, Amtsblatt RS, No. 54/92, zuletzt geändert 2006. 
Ausgangspunkt bei der Regelung der arbeits- und sozialrechtlichen Stellung eines Beamten ist die Verknüpfung zweier Rechtsgebiete. Sonderbestimmungen können unter Umständen zum Schutz des öffentlichen Interesses gerechtfertigt sein, dürfen aber nicht nur auf das übertragene Amt abstellen, sondern müssen die tatsächlichen besonderen Gegebenheiten der Dienstausübung berücksichtigen und die Verhältnismäßigkeit im Vergleich mit den Regelungen anderer Arbeitnehmer wahren. Die Errichtung eines eigenständigen (nicht beitragspflichtigen) Alterssicherungssystems für Beamte kann mit verfassungsrechtlichen Werten kollidieren, die ihren Ausdruck in dem Sozialstaatsprinzip, dem Gleichheitsgrundsatz und dem Recht auf soziale Sicherheit finden. Ein solches System oder über ein allgemeines Maß hinausgehende Leistungen stünden eher im Widerspruch zum öffentlichen Interesse anstatt es zu schützen.

Sonderbestimmungen für bestimmte Gruppen von Beamten sind insbesondere in der gesetzlichen Rentenversicherung vorgesehen. Diese Regelungen in einem einzigen Gesetz zusammenzufassen, würde größere Transparenz bieten. Die obligatorische Rentenzusatzversicherung erfaßt Beschäftigungen sowohl im privaten wie im öffentlichen Sektor, ohne große Unterschiede zu machen. Die kollektive Zusatzversicherung für Beamte ist der freiwilligen Rentenversicherung im privaten Sektor sehr ähnlich. Einer der Unterschiede ist, daß die Entscheidung, die Zusatzversicherung zu begründen, überwiegend auf kollektiver statt auf individueller Ebene getroffen wurde.

Die Gleichbehandlung aller Erwerbstätigen hat grundsätzlich Vorteile. Sicherlich bestehen Unterschiede in der Wahrnehmung eines „separaten“ gegenüber einem ,allgemeinen" Rentenversicherungssystem. Doch bieten ein breiteres Solidaritätsnetz, weniger Verwaltungsaufwand, mehr Transparenz und geringere Hürden beim Wechsel von einem Sektor in den anderen deutliche Vorzüge.

Darüber hinaus ist die Durchführung von Rentenreformen einfacher, wenn Beamte in der allgemeinen Rentenversicherung versichert sind. In Slowenien wurde die letzte Rentenreform im Jahr 2000 durchgeführt. Die Übergangsphase ist zwar noch nicht abgeschlossen, doch sind bereits neue Reformvorschläge erarbeitet. Die ständigen Entwicklungen der sozialen Gegebenheiten und Verhältnisse erfordern auch eine Veränderung der sozialen Sicherheitssysteme einschließlich der gesetzlichen Rentenversicherung.

Die Kernfrage ist nicht ob, sondern wie die gesetzliche Rentenversicherung de lege ferenda modifiziert werden soll. Die gegenwärtige Entwicklung sollte beibehalten werden, insbesondere sollte die gesamte Erwerbsphase zur Grundlage der Rentenberechnung gemacht werden. In politischer Hinsicht mag es reizvoll erscheinen, neue Sicherungssysteme zu erarbeiten, wenn das bestehende Schwierigkeiten bereitet. Fraglich ist, ob durch diese Vorgehensweise bestehenden Herausforderungen besser begegnet werden kann. Denn wenn das bestehende Modell nicht erfolgreich durchgeführt werden kann, heißt das nicht, daß das neue Modell automatisch besser funktionieren wird.

Moderne Ansätze, die gesetzliche Rentenversicherung zu modifizieren, scheinen vielmehr eine Rechtfertigung dafür zu sein, mehr privatrechtliche Komponenten (wie fiktive Rentenkonten) zum Nachteil des Solidaritätsprinzips einzuführen. 
Unabhängig davon, welcher Weg gewählt wird, muß die soziale Sicherheit so ausgestaltet werden, daß sie ihr Ziel erreicht, nämlich ungeachtet des jeweiligen (öffentlichen oder privaten) Sektors, des Geschlechts, der Einkünfte, des Vermögens oder anderer persönlicher Verhältnisse eine angemessene Einkommenssicherung im Alter zu gewährleisten. 


\title{
Die Altersversorgung von Beamten in Tschechien
}

\author{
Martin Štefko
}

\section{Einleitung}

II. Alterssicherungssysteme für Beschäftigte im öffentlichen Dienst

1. Das allgemeine Rentenversicherungssystem

2. Besondere Regelungen für Staatsbedienstete

III. Bewertung 